\title{
Regression of Dural Arteriovenous Fistulae after Venous Flow Reconstructive Surgery in a Case with Hemangiopericytoma at the Confluence of Sinuses
}

\author{
Kota Kurisu Hiroaki Motegi Toshiya Osanai Hiroyuki Kobayashi \\ Shunsuke Terasaka Kiyohiro Houkin \\ Department of Neurosurgery, Graduate School of Medicine, Hokkaido University, \\ Sapporo, Japan
}

\section{Key Words}

Confluence of sinuses - Dural arteriovenous fistula - Hemangiopericytoma - Sinus occlusion .

Venous hypertension - Venous reconstructive surgery

\begin{abstract}
Background: The mechanism by which acquired dural arteriovenous fistula (dAVF) develops is still unclear. Few cases have been reported with both dAVF and intracranial tumors, and in these few cases the authors have proposed that induced venous hypertension may lead to the pathogenesis of dAVF. We experienced a case of intrasinusoidal hemangiopericytoma (HPC) with dAVF development. In addition to its rare pathology and tumor location, this case showed regression of dAVF immediately after tumor removal. Case Report: The patient was a 23-year-old man who developed progressively worse headaches and papilledema. The HPC was located entirely inside the confluence of the sinuses $(C O S)$ and resulted in venous sinus occlusion. Cerebral angiography demonstrated a dAVF located in the straight sinus, upstream of the occluded CoS, which was fed by the dural branch of the posterior cerebral artery. After the endovascular embolization of the tumor feeders, subsequent surgery included venous reconstruction in addition to tumor excision. Although the dAVF was not treated with an endovascular procedure or surgery, postoperative angiography revealed complete disappearance of the dAVF. Conclusion: We conclude that venous reconstructive surgery greatly contributed to the immediate regression of the dAVF. When planning the treatment strategy for such cases, it should be remembered that acquired dAVF may regress due to the normalization of venous hypertension.

(c) 2014 S. Karger AG, Basel
\end{abstract}

Shunsuke Terasaka, MD

Department of Neurosurgery

Graduate School of Medicine, Hokkaido University

North 15, West 7, Kita-ku, Sapporo 060-8638 (Japan)

E-Mail terasas@med.hokudai.ac.jp 
Kurisu et al.: Regression of Dural Arteriovenous Fistulae after Venous Flow Reconstructive Surgery in a Case with HPC at the Confluence of Sinuses

\section{Introduction}

Dural arteriovenous fistula (dAVF) is characterized by abnormal shunts of blood flow within the dural leaflets. Although most dAVFs are considered to be acquired lesions [1, 2], the underlying mechanism controlling how and why dAVFs develop is unclear. It has long been speculated that various factors contribute to the formation of dAVF: sinus thrombosis, sinus hypertension, intracranial abscess, and trauma among others. Since Djindjian and Merland [3] first noted an association between dural sinus occlusion and dAVF development in 1978, some cases have been reported $[1,4-8]$ in which the sinus occlusion by the intracranial tumor initiated development of dAVF. We experienced a case of dAVF associated with hemangiopericytoma (HPC) that was located entirely inside the confluence of the sinuses $(\mathrm{CoS})$ and resulted in venous sinus occlusion. After tumor removal with venous flow reconstruction, the dAVF fed by the posterior cerebral artery (PCA) showed absolute disappearance, despite the fact that this lesion was not treated with an endovascular procedure or surgery.

Based on the clinical findings of this case, we discuss the mechanism of dAVF development.

\section{Case Report}

A 23-year-old man with a 6-month history of progressive headache was admitted to our institution. On physical examination, mild bilateral papilledema was apparent. Magnetic resonance (MR) imaging of the brain disclosed the existence of a round extra-axial tumor within the $\operatorname{CoS}$ (fig. 1a-c). The lesion appeared slightly hyperintense compared to the gray matter on both T1- and T2-weighted sequences, and prominent intratumoral flow voids were observed on a T2-weighted image (fig. 1a). There was no surrounding brain edema or hyperostosis. A contrast-enhanced T1-weighted image (fig. 1b, c) revealed a homogenous enhancement, and the observed cap sign consisted of the tumor and venous blood in the sagittal view. The tumor was thought to have originated from the inside wall of the dural sinus and expanded toward the lumen of the CoS.

Both carotid angiograms revealed occlusion of the CoS by the occupying tumor (fig. 2a). In both external carotid angiograms, moderate tumor stain feeding from both occipital arteries, both middle meningeal arteries (MMAs), and the right ascending pharyngeal artery was observed (fig. 2b). A vertebral angiogram (fig. 2c, d) showed an abnormal early filling of the straight sinus via the enlarged and tortuous dural branch of the left PCA, the so-called Davidoff-Schechter artery, which we diagnosed as dAVF. The shunt drained into the straight sinus in a retrograde fashion. There was no apparent tumor staining from both external carotid angiograms. Thus, the shunt fed by the left PCA was considered to be directly connected to the straight sinus, not through the tumor. A sagittal view of cone beam CT showed that the enlarged Davidoff-Schechter artery directly connected to the straight sinus. The exact location of the fistulous point was observed just ventral to the tumor without tumor stain (fig. 2e). We planned transarterial embolization of tumor feeders before the surgery for tumor excision.

For bilateral occipital arteries, coil embolization with tornado microcoils (Cook, Bloomington, India) was performed; for bilateral MMAs and the right ascending pharyngeal artery, N-butyl cyanoacrylate (NBCA) was used for the embolization of feeding arteries. 25\% NBCA for the left MMA, 33\% NBCA for the right ascending pharyngeal artery, and 50\% NBCA for the right MMA was used, respectively. At the end of the endovascular procedure, angiograms 
Kurisu et al.: Regression of Dural Arteriovenous Fistulae after Venous Flow Reconstructive Surgery in a Case with HPC at the Confluence of Sinuses

from external carotid arteries suggested good embolization of feeding arteries (fig. 2f). The dAVF fed by the PCA was untreated because of technical difficulty and associated high risk.

Three days after the endovascular treatment, the patient underwent surgical excision of the tumor. After bilateral occipital and suboccipital craniotomy, the superior sagittal sinus, the $\mathrm{CoS}$, and the bilateral transverse sinuses were exposed. The tumor was located entirely inside the dural sinus. The wall of the CoS was incised and tumor resection continued until profuse bleeding from the opened dural sinuses was encountered. The bleeding was controlled by placement of pliable patties with strings. Because the tumor did not invade the anterior wall of the sinus, a gross total resection of the tumor was accomplished. Subsequently, the sinus was repaired using an artificial dura mater of Gore-Tex membrane (WL Gore \& Associates, Inc., USA), and venous flow restoration was accomplished.

The pathological finding of a spindle-shaped hypercellular neoplasm with numerous vascular channels was consistent with HPC. Postoperatively, the patient showed complete resolution of the headache and papilledema. MR imaging demonstrated no residual lesion (fig. 3a), and postoperative angiograms 1 week after the surgery demonstrated the restoration of anterograde venous flow through the repaired CoS (fig. 3b). The dAVF fed by the PCA had completely disappeared, although this lesion was not treated with an endovascular procedure or surgery (fig. 3c). The patient was discharged home uneventfully and had been free from disease for 14 months after surgery.

\section{Discussion}

This report presented a case of dAVF associated with HPC that was located entirely inside the dural sinus of the CoS. To the best of our knowledge, there has only been one case report that described HPC located entirely inside the CoS [9]. This extremely rare location of the tumor resulted in dural sinus occlusion. Although the association of dAVF and sinus occlusion induced by intracranial tumors is reported by several authors $[1,4-8]$, there have only been two case reports that describe the cases with HPCs $[4,8]$.

Though the definitive underlying mechanism by which dAVF develops secondary to intracranial tumors is still unclear, several authors have proposed possible theories of dAVF formation. One possible mechanism is that induced venous hypertension by sinus obstruction may inflict physical stress to adjacent vasculature [5, 7]. Sustained venous hypertension within the sinuses will be transmitted in a retrograde fashion to the capillaries and arterioles [10]. Then, the vessels dilate, the sphincters become incompetent, and a dAVF develops. Based on an animal experiment, Terada et al. [10] reported that a new formation of AVFs was found in 13.6-23.1\% of rats in which chronic venous hypertension was surgically induced.

A second possible mechanism for the formation of dAVF is the association of angiogenic factors, such as vascular endothelial growth factor and basic fibroblast growth factor, among others. Tissue hypoxia as a result of venous congestion [11], increased intraluminal pressure in vessels [12], and the tumor itself [13] may stimulate or release angiogenic factors. The stimulated angiogenic factors may develop new vessels in the sinus or venous wall and induce the formation of AVFs. In a different rodent model of dAVF, Shin et al. [14] proved the hyperexpression of vascular endothelial growth factor in the wall of the dura mater and in addition to neuron cells of the brain parenchyma.

Another considered mechanism is that venous hypertension may induce enlargement of the microscopic AVFs that normally exist between arteries and veins in the dura mater at the site of the dural sinuses $[10,15]$. Although several possible mechanisms have been 
proposed, we speculated that microscopic AVFs largely contributed to the pathogenesis of $\mathrm{dAVF}$ in this case. The phenomenon of the rapid regression of the dAVF within a week after normalization of the venous hypertension strongly supports this theory.

To cure dAVF associated with intracranial tumor, several authors [4-6] have suggested additional surgery $[4,5]$ or an endovascular approach $[5,6]$ to tumor resection surgery. There is only one other case report [7] in which dAVF also disappeared after tumor resection without any additional treatment. In contrast to our presented case, partial tumor removal without venous reconstruction was performed and regression of the dAVF was observed 9 months after operation [7]. All reported cases [4-6] that only underwent tumor resection surgery required additional procedures for the associated dAVF. We emphasize that venous reconstruction leading to the immediate resolution of venous hypertension was very effective for the regression of the dAVF. Supporting our speculation, the phenomenon of $\mathrm{dAVF}$ regression is also observed after the normalization of venous pressure in animal experiments [10].

\section{Conclusion}

In a case of $\mathrm{dAVF}$ associated with intracranial tumor, induced venous hypertension could act as a trigger for dAVF development. When planning a treatment strategy for such cases, we should take into consideration that regression of associated dAVF may occur by normalization of venous hypertension.

\section{Disclosure Statement}

This study received no funding and the authors declare no conflicts of interest.

\section{References}

1 Ahn JY, Lee BH, Cho YJ, Joo JY, Lee KS: Dural arteriovenous fistula associated with meningioma: spontaneous disappearance after tumor removal. Neurol Med Chir 2003;43:308-311.

2 Chaudhary MY, Sachdev VP, Cho SH, Weitzner I Jr, Puljic S, Huang YP: Dural arteriovenous malformation of the major venous sinuses: an acquired lesion. AJNR Am J Neuroradiol 1982;3:13-19.

-3 Djindjian R, Merland J: Meningeal Arteriovenous Fistulae. Super-Selective Arteriography of the External Carotid Artery. Berlin, Springer, 1978.

-4 Kalani MY, Martirosyan NL, Eschbacher JM, Nakaji P, Albuquerque FC, Spetzler RF: Large hemangiopericytoma associated with arteriovenous malformations and dural arteriovenous fistulae. World Neurosurg 2011;76:592e7-10.

5 Vilela P, Willinsky R, terBrugge K: Dural arteriovenous fistula associated with neoplastic dural sinus thrombosis: two cases. Neuroradiology 2001;43:816-820.

6 Toledo MM, Wilson TJ, Dashti S, McDougall CG, Spetzler RF: Dural arteriovenous fistula associated with superior sagittal sinus occlusion secondary to invasion by a parafalcine meningioma: case report. Neurosurgery 2010;67:205-207.

7 Horinaka N, Nonaka Y, Nakayama T, Mori K, Wada R, Maeda M: Dural arteriovenous fistula of the transverse sinus with concomitant ipsilateral meningioma. Acta Neurochir 2003;145:501-504.

-8 Bitoh S, Hasegawa H, Kato A, Tamura K, Mabuchi E, Kobayashi Y: Meningeal neoplasms associated with cerebral vascular malformations. Surg Neurol 1987;27:469-475.

-9 Collignon FP, Cohen-Gadol AA, Piepgras DG: Hemangiopericytoma of the confluence of sinuses and the transverse sinuses. Case report. J Neurosurg 2003;99:1085-1088.

-10 Terada T, Higashida RT, Halbach VV, Dowd CF, Tsuura M, Komai N, Wilson CB, Hieshima GB: Development of acquired arteriovenous fistulas in rats due to venous hypertension. J Neurosurg 1994;80:884-889. 
Kurisu et al.: Regression of Dural Arteriovenous Fistulae after Venous Flow Reconstructive Surgery in a Case with HPC at the Confluence of Sinuses

11 Wiesener MS, Turley H, Allen WE, Willam C, Eckardt KU, Talks KL, Wood SM, Gatter KC, Harris AL, Pugh CW, Ratcliffe PJ, Maxwell PH: Induction of endothelial PAS domain protein-1 by hypoxia: characterization and comparison with hypoxia-inducible factor-1alpha. Blood 1998;92:2260-2268.

-12 Miano JM, Vlasic N, Tota RR, Stemerman MB: Smooth muscle cell immediate-early gene and growth factor activation follows vascular injury. A putative in vivo mechanism for autocrine growth. Arterioscler Thromb 1993;13:211-219.

13 Dietzmann K, von Bossanyi P, Warich-Kirches M, Kirches E, Synowitz HJ, Firsching R: Immunohistochemical detection of vascular growth factors in angiomatous and atypical meningiomas, as well as hemangiopericytomas. Pathol Res Pract 1997;193:503-510.

14 Shin Y, Uranishi R, Nakase H, Sakaki T: Vascular endothelial growth factor expression in the rat dural arteriovenous fistula model. No To Shinkei 2003;55:946-952.

-15 Kerber CW, Newton TH: The macro and microvasculature of the dura mater. Neuroradiology 1973;6:175179.
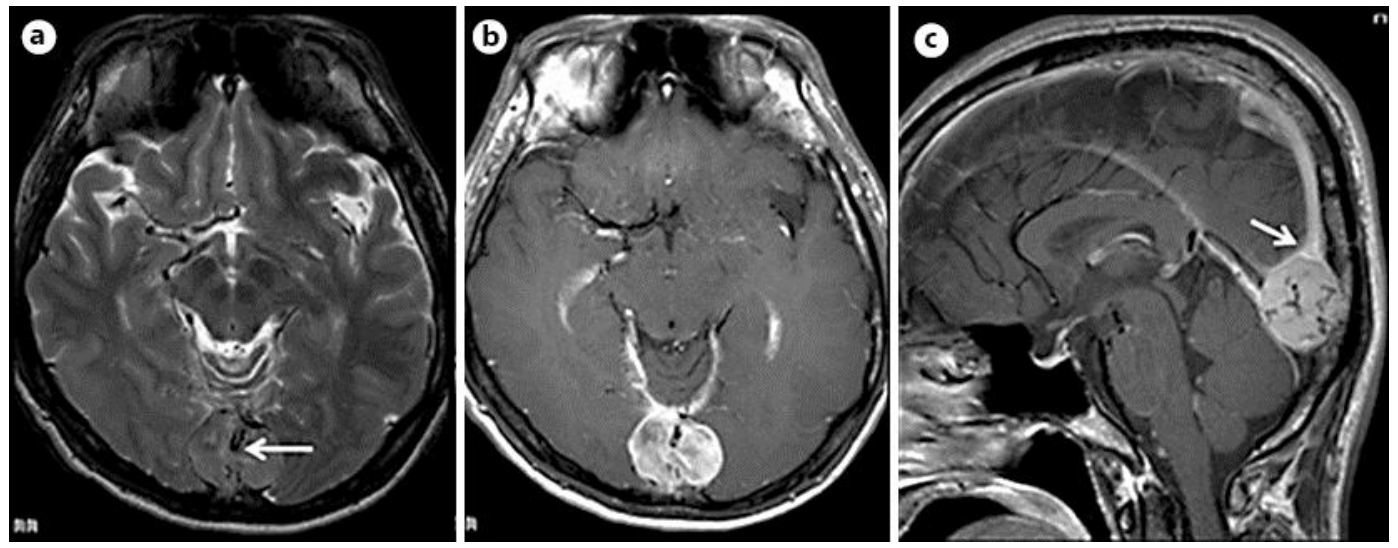

Fig. 1. a Axial T2-weighted MR image showing a round extra-axial tumor within the CoS. The lesion appears slightly hyperintense compared with the gray matter. Prominent intratumoral flow voids are observed (arrow). b Axial contrast-enhanced T1-weighted image revealing a homogenous strong enhancement of the tumor without a dural tail sign. c Sagittal contrast-enhanced T1-weighted image demonstrating a 'cap sign' consisting of the tumor and venous blood (arrow), which indicates that the tumor originated from inside of the CoS. 


\section{Case Reports in Neurology}
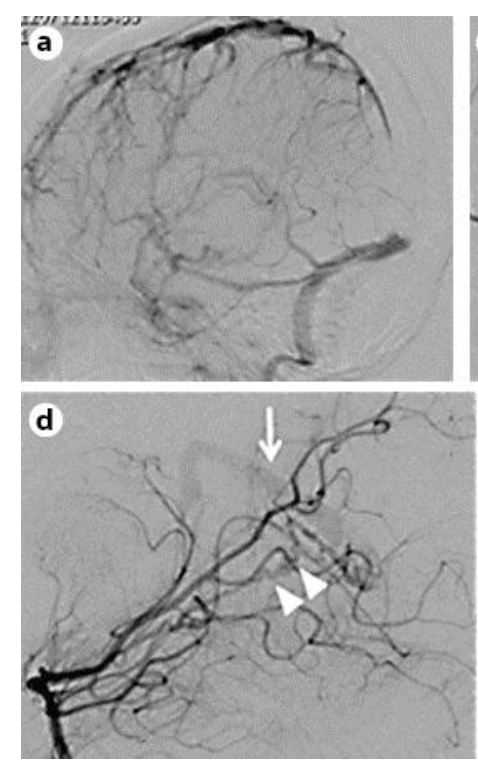

\begin{tabular}{l|l}
\hline \multicolumn{2}{l}{ Case Rep Neurol 2014;6:207-212 } \\
\hline DOI: $10.1159 / 000365882$ & $\begin{array}{l}\text { C 2014 S. Karger AG, Basel } \\
\text { www.karger.com/crn }\end{array}$ \\
\hline
\end{tabular}

Kurisu et al.: Regression of Dural Arteriovenous Fistulae after Venous Flow Reconstructive Surgery in a Case with HPC at the Confluence of Sinuses
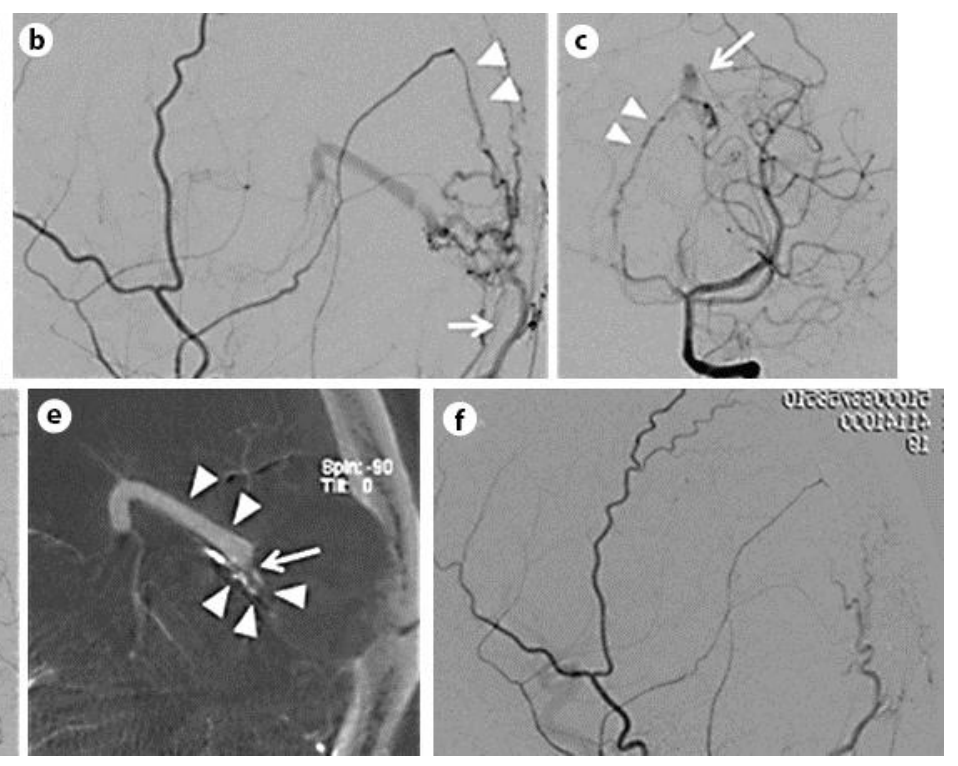

Fig. 2. a Carotid angiogram revealing the occlusion of the CoS by the tumor. $\mathbf{b}$ In a left external carotid angiogram, moderate tumor stain feeding from the occipital artery (arrow) and MMA (arrow heads) was observed. Anteroposterior view (c) and lateral view (d) of vertebral angiograms showing an abnormal early filling of the straight sinus (arrow) via an enlarged and tortuous dural branch of the left PCA (arrow heads) without any tumor stain, which we diagnosed as the dAVF. e Cone beam CT vertebral angiogram clearly depicted the route of the AVF and fistulous point. The exact location of the fistulous point (arrow) was observed just ventral to the tumor without tumor stain. $f$ After the endovascular procedure, the lateral view of an angiogram from external carotid arteries revealed good embolization of tumor suppliers.
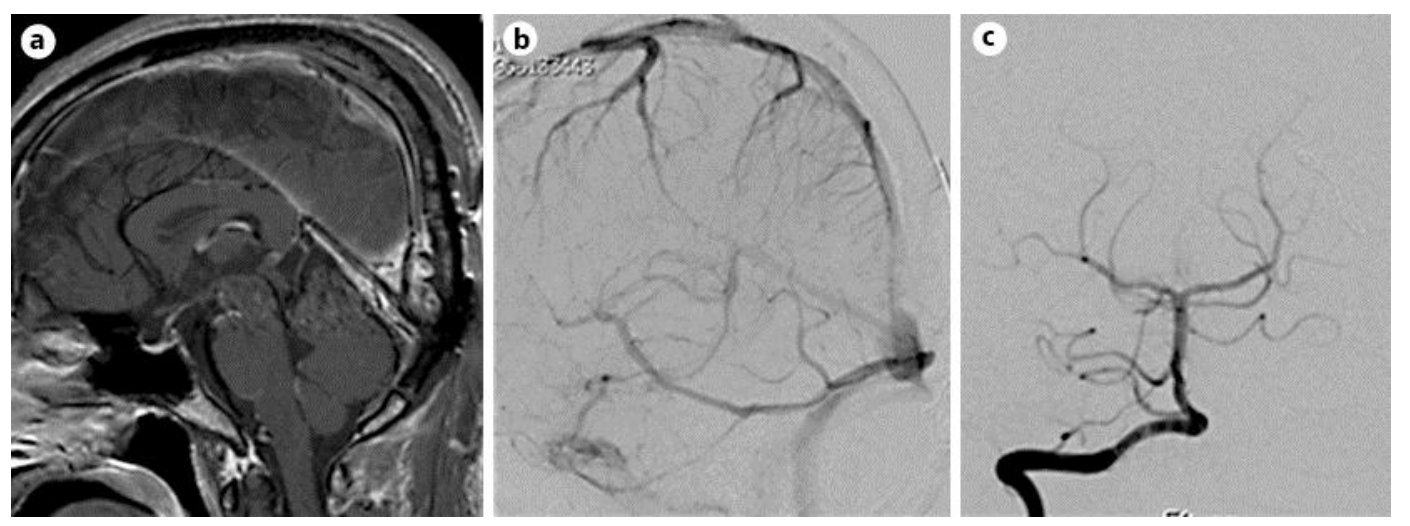

Fig. 3. a Postoperative MR image confirming the gross total resection of the tumor. $\mathbf{b}$ Lateral view of a carotid angiogram demonstrating restored venous flow at the CoS. c Anteroposterior view of a vertebral angiogram revealing complete regression of the dAVF that was observed preoperatively. 